\title{
Phylogenetic analysis of canine parvoviruses from Turkey
}

\author{
ZEYNEP AKKUTAY-YOLDAR, B. TAYLAN KOÇ*
}

\begin{abstract}
Ankara University, Faculty of Veterinary Medicine, Department of Virology, Diskapi 06110, Ankara, Turkey *Aydın Adnan Menderes University, Faculty of Veterinary Medicine, Department of Virology, 09016, Aydın, Turkey
\end{abstract}

\section{Akkutay-Yoldar Z., Koç B. T. \\ Phylogenetic analysis of canine parvoviruses from Turkey}

Summary

Canine parvovirus (CPV) type 2 is the causative agent of acute hemorrhagic enteritis and high mortality in the affected dogs. Numerous studies have been done to understand the origin of the virus and to exhibit new variants and circulating strains. This report describes the detection and genomic characterization of CPV strains from indoor and outdoor dogs in Ankara, Turkey. Samples were sent to our laboratory due to clinical symptoms in puppies. We tested blood and swab samples to determine the presence of canine parvovirus (CPV) in three puppies and two adult dogs by reverse transcription-polymerase chain reaction (RT-PCR) using VP2 (capsid protein) region primers of canine parvoviruses. Following that, to provide molecular characterization data Maximum Likelihood (ML) method was used for phylogenetic analyses. Constructed phylogenetic trees from the aligned nucleotide sequences revealed that our CPV strains demonstrated high genetic similarities, with $100 \%$ identity match on nucleotide alignments with each other and classified in CPV-2b genotypes.They have placed on a monophyletic clade as a sister branch with CPV VAC S quantum with 98.9\% nucleotide homology. Our findings suggest that CPV-2b is actual and frequently seen variant in Turkey and shows high similarities with other CPV variants and a bit less with FPVs in Turkey and around the world. CPV causes high mortality and morbidity in dogs and to develop effective vaccines for protection of dogs in Turkey where there are few numbers of studies that have been done, field strains should be isolated and characterised.

Keywords: canine parvovirus, dog, molecular characterization, PCR, phylogenetic analysis

Carnivore protoparvovirus 1, known as Canine parvovirus (CPV), belongs to the genus Protoparvovirus under the subfamily Parvovirinae and the family Parvoviridae. It causes a highly contagious and fatal disease in dogs (ICTV 2018) and is genetically and antigenically different from CPV-1 (canine minute virus), responsible for neonatal death in dogs as well as involved in the Bocavirus genus (34). Canine parvovirus, the enteric pathogen of dogs, has four main antigenic types: CPV-2, CPV-2a, CPV-2b and CPV-2c (24). They are single-stranded, negative-sense DNA viruses, with a $26 \mathrm{~nm}$ diameter icosahedral capsid that is non-enveloped and consists of VP1 and VP2 viral proteins (27). The genome is around $5 \mathrm{~kb}$ in length with two open reading frames (ORFs); the first encodes the non-structural proteins NS1 and NS2, and the second encodes the VP1 and VP2 structural capsid proteins (27). VP2 takes on a significant function in the host immune response (24).

Canine parvoviruses first emerged among dogs in 1978 and have caused the deaths of an excessive num- ber of dogs around the world (2). CPV-2 evolved and three other variants have occurred, two of them (CPV2a, CPV-2b) are similar, but one (CPV-2c) has a unique antigenic pattern, according to CPV-2 (3). Now, the most recent variant $\mathrm{CPV}-2 \mathrm{c}$ has caused morbidity and mortality in dogs around the world $(3,4,9,19)$. CPV-2c was first described in Italy following its occurrence in leopard cats in Vietnam and then in Europe $(11,18)$. Today, all three variants can cause severe clinical signs, such as lifelong cardiac disease, leucopenia, pyrexia, haemorrhagic diarrhoea, myocarditis and death in dogs. It disseminates in many countries $(5,20,25,36,37)$.

$\mathrm{CPV}$ is transmitted through the oronasal route with the virus in faeces. Three to seven days following infection, the virus is shed in the faeces and clinical symptoms occur, as mentioned, in young dogs; adult dogs can stay asymptomatic with the help of immunity gained through vaccination or previous transmissions $(1,5,8)$.

It is thought that the virus originates from the feline panleukopenia virus (FPV) via minor mutations in the 
capsid protein and only these few substitutions differentiate CPV-2 from the other antigenic types, FPV and mink enteritis virus (MEV) $(24,36)$. After mutations, the CPV-2 variants that have a high rate of evolution compared to FPV have become able to infect both dogs and cats and have been found in wolves, foxes, other canids and felids $(14,15,32)$.

CVP-2 has been previously reported in cats and dogs in Turkey, so obtaining up to date information representing the circulation of CPV variants is a necessity $(22,28,35,39)$. The objective of this study was to determine the CPV variants in pups with clinical symptoms and to demonstrate its relationship with the other types reported in Turkey and around the world.

\section{Material and methods}

Specimens. Blood samples from three dogs, and blood and swab samples from two different dogs were sent for laboratory diagnosis. CPV/TR/2018/Y1, CPV/TR/2018/Y2 and CPV/TR/2018/Y3 were homeless puppies. CPV/TR/ 2018/serce and CPV/TR/2018/efe were indoor puppies. Typical clinical signs of canine parvoviruses, such as acute enteritis, anorexia, depression, high body temperature and vomiting, were observed in all sampled animals.

Polymerase Chain Reaction (PCR) and phylogenetic analysis. Samples were used for total DNA extraction according to the phenol: chloroform: isoamyl alcohol extraction method described by Sambrook and Russell (30). The DNA pellets were resuspended with $20 \mu 1$ of deionised water and used as a template for polymerase chain reaction (PCR) applications. All DNA was stored at $-20^{\circ} \mathrm{C}$ until testing. PCR for partial genes was performed using oligonucleotide $\mathrm{Pb}$ sense and anti-sense primers (5'-CTTTAACCTTCCTGTAACAG-3' 4043-4062 and 5'-CATAGTTAAATTGGTTATCTAC-3' 4449-4470) targeting a 427-bp fragment of the VP2 gene of the parvovirus, as described by Pereria et al. (25). This was carried out in a total volume of $30 \mu \mathrm{l}$ of mix containing $5 \mathrm{U} / \mu 1 \mathrm{Taq}$ DNA polymerase (MBI, Fermentas, Waltham, MA, USA), $10 \times$ Taq Buffer, primers and $3 \mu \mathrm{l}$ of extracted DNA. The thermocycler was programmed with the following steps and cycling times: $95^{\circ} \mathrm{C}$ for $10 \mathrm{~min}$, followed by 40 cycles of $95^{\circ} \mathrm{C}$ for $30 \mathrm{~s}$, $50^{\circ} \mathrm{C}$ for $30 \mathrm{~s}, 72^{\circ} \mathrm{C}$ for $60 \mathrm{~s}$ and a final extension stage of $72^{\circ} \mathrm{C}$ for $10 \mathrm{~min}$.

The obtained amplicons were purified using a Gene JET PCR purification Kit and sent to BM Labosis for Sanger sequencing. Phylogeographic analysis of the partial VP2 region targeting a $325 \mathrm{bp}$ region was performed using 101 canine and feline parvovirus sequences from Turkey and different geographical regions of the world, available in GenBank. The raw sequence reads were analysed and assembled, and the obtained sequences were aligned by using CLC Main Workbench v5.5. To interpret the results of the phylogeographic analysis, an SDT a stand-alone program was used (Fig. 2) (21). A graphical port demonstrated pairwise identity scores using a colour-coded pairwise-identity matrix and enabled the visualisation of the proximity among sequences in a dataset. To obtain more accurate result in a larger area, a second phylogenetic analysis was performed with reference strains targeting a $378 \mathrm{bp}$ region. Due to their short sequences, gene regions from Turkey have not been included in this analysis. Phylogenetic analyses were performed with the help of the software program MEGA $X$ (17). Maximum likelihood trees were constructed based on the Kimura two-parameter model with 1,000 bootstrap replicates for the determination of genetic distances between nucleotide sequences (Fig. 1). GenBank accession numbers for the VP2 gene of the CPV sequences presented in this article are: MK248872, MK248873, MK248874, MK248875, MK248876, MK248877 and MK248878 for CPV/TR/2018/ serçe/k CPV/TR/2018/efe/k, CPV/TR/2018/serçe/s, CPV/ TR/2018/efe/s, CPV/TR/2018/Y1, CPV/TR/2018/Y2 and CPV/TR/2018/Y3, respectively. In order to distinguish two samples taken from the same dog; the letters ,k" and „s" represent blood and swab samples, respectively.

\section{Results and discussion}

The specific amplicon of the $427 \mathrm{bp}$ DNA product was amplified by PCR from all the samples using $\mathrm{Pb}$ sense and anti-sense primers, showing that these puppies were infected by canine parvovirus. After trimming and editing processes of the low-quality nucleotides in relevant sequences, nucleotide similarity searches by the Basic Local Alignment Search Tool (BLAST) revealed that the sequences of the blood and rectal swab samples were 98.42-99.47\% identical to various CPV and FPV strains. Phylogeographic analysis of the $325 \mathrm{bp}$ nucleotide sequence of the field virus with several canine and feline parvovirus sequences revealed that the obtained sequences were located inside the canine parvovirus strains with an FPV strain (HM042740.1) isolated from a cat and reported by Muz et al. (22) (Fig. 1). TR-URF/CPV-2b/ (MG780292) located in this clade placed as a distinct branch with the seven field strains (Fig. 1).

By using the SDT program approximately 100 virus sequence identities were established. Pairwise identity changed between the interval of 100 to $88 \%$ and this was shown in colours: from maroon, dark red, red, orange-red, orange, yellow, yellow-green, aquamarine, turquoise, blue to navy. The CPV strains reported in this study largely gave a maroon ( $99-100 \%)$ and red colour ( $\sim 99 \%)$ among its kind but gave orange-red ( $\sim 98 \%)$ with some and yellow ( $95 \%)$ with one of the $2 b$ strains submitted from the Urfa province of Turkey. Urfa $2 b$ strains also showed high divergence with the CPV and FPV strains reported from Turkey and the world, demonstrating mostly yellow $(\sim 95 \%)$, some turquoise ( $\sim 91-92 \%)$ and yellow-turquoise-blue $(\sim 89-95 \%)$ colours, respectively. In the overall SDT graphic, some FPV and Urfa $2 \mathrm{~b}$ strains exhibited the highest diversity, appearing as navy ( $88 \%)$.

In the second phylogenetic analysis for the $378 \mathrm{bp}$ partial VP2 gene (Fig. 3), sequence homology results showed that the field CPV strains shared 100\% nucleotide identity and $99.5 \%$ similarity rates with each other (Fig. 4). The CPV vaccine strain S quan- 


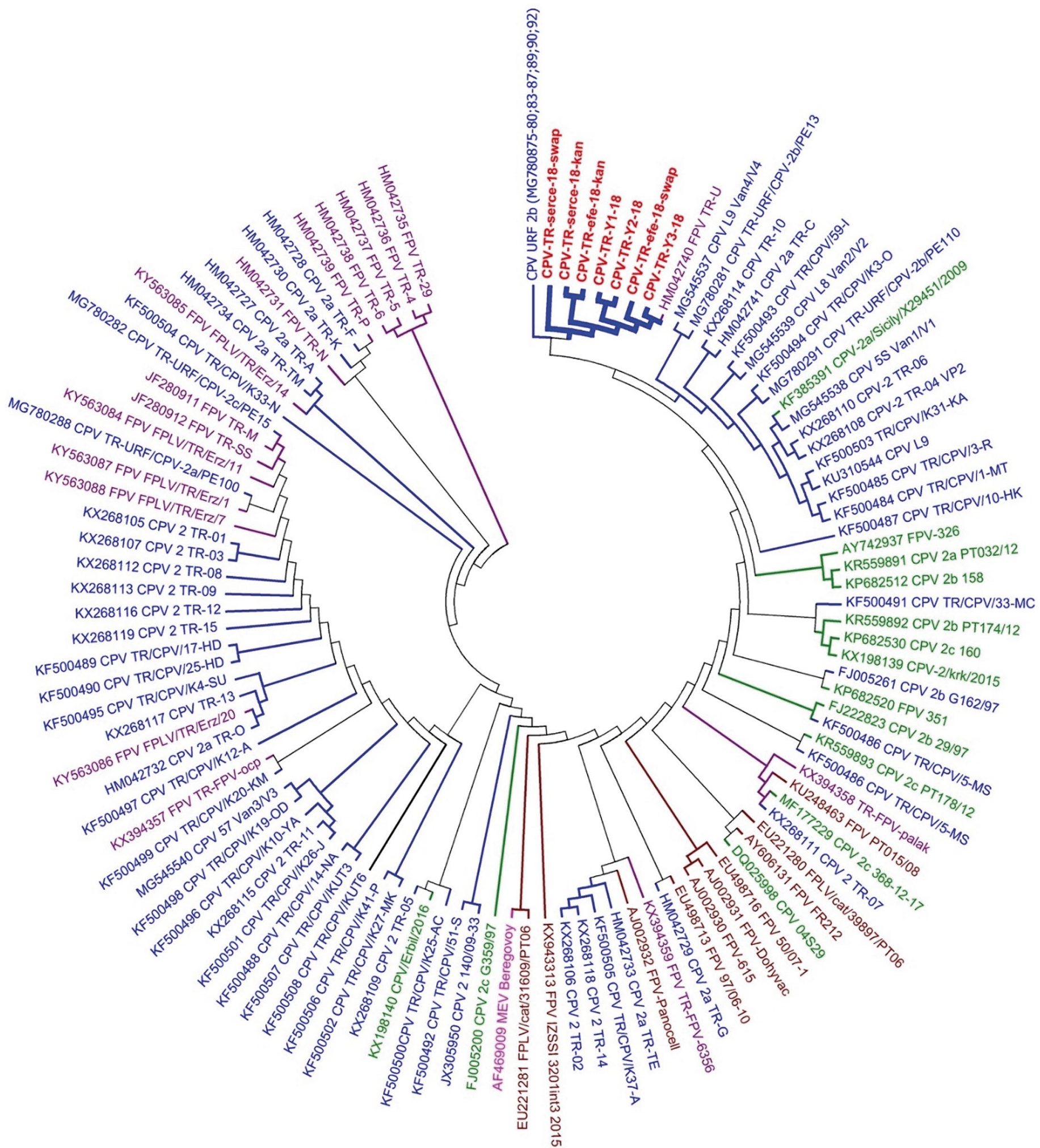

Fig. 1. Phylogenetic tree constructed based on the fragments 325 nucleotides of VP2 gene region, showing the relationship of CPV and FPV strains by Maximum Likelihood method

tum, obtained from Thailand in 2009, had a nucleotide identity of $98.9 \%$ with all seven field strains and was localised on a monophyletic clade as a sister branch. Our field strains compromised a clade with CPV VAC $\mathrm{S}$ quantum and were found to be close to the CPV-2b group. CPV/Jiangsu/, isolated from China in 2012, CPV/42/ from Italy in 2005 and CPV/04S23/, detected in France in 2005, clustered together by representing the CPV-2b group. All three CPV-2b sequences had a nucleotide identity of $98.9 \%$ with all seven field strains, as well as the other CPV-2b strain: CPV VAC $\mathrm{S}$ quantum.

Considering the proximity to the $2 \mathrm{c}$ group, the strains obtained in this study demonstrated $98.7 \%$ similarity with CPV/136/ from Italy, obtained in 2000, and 98.9\% nucleotide similarity with the rest of the group, consist- 

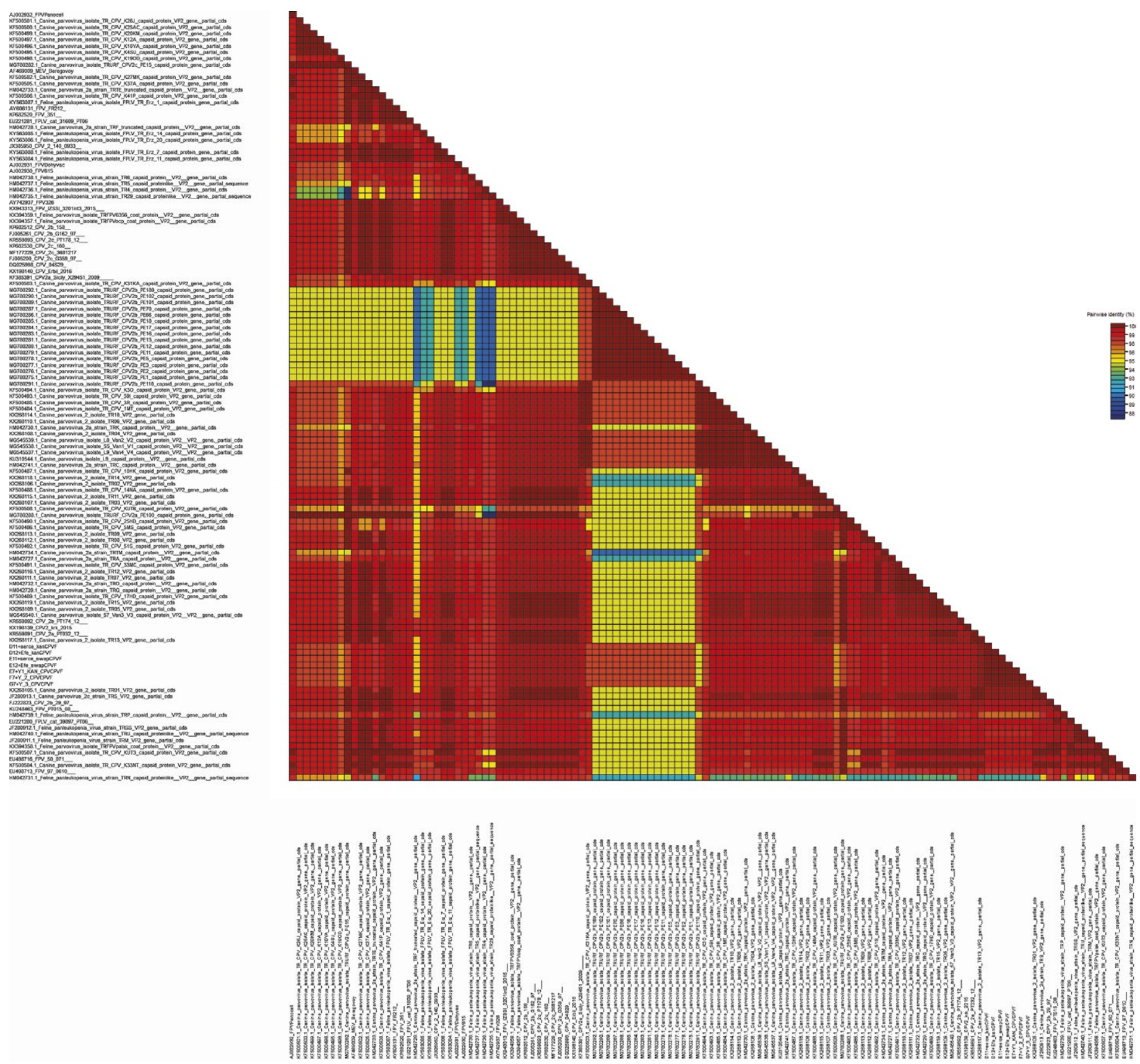

Fig. 2. SDT color-coded matrix of pairwise identity scores generated by alignment of a 325 bp fragment $3 D$ gene for 101 viruses. Each colored square symbolizes a percentage identity score between two sequences (one indicated horizontally to the left and the other vertically at the bottom)

ing of CPV/G133/97/FJ005198 isolated in Germany in 1997, CPV/GR51/ reported from Greece in 2008, $\mathrm{CPV} / 67 /$ detected in the USA in 2007 and CPV/ Parana/ reported in Brazil in 2009.

All the strains obtained in this study showed $98.9 \%$ nucleotide homology with the 2a group strains CPV $12 / 08-B / G U 362934$ isolated in Italy in 2008 and CPV/04S17 obtained from the field in France in 2005, and $98.7 \%$ similarity with CPV/PassoFundo/ obtained in Brazil in 2009. They also exhibited 98.4\% nucleotide homology to vaccine strains: CPV/Intervet/VP2 gene cloned in 2006 by Intervet and CPV/Merial/VP2 gene cloned in 2006 by Merial. Two strains shared 100\% nucleotide identities and mapped phylogenetically to the same branch.
When the proximity between the CPV strains obtained in this study and FPV strains was examined, similar nucleotide variations were observed with a small decrease with the FPV vaccine strains; these had $98.2 \%$ sequence homology with the FPV/Purevax/ vaccine strain detected in 2008, and FPV/Felocell/, the Pfizer vaccine strain characterised in 2008, which mapped phylogenetically to the same branch. FPV/ EU018144 obtained in Argentina in 2007 also displayed $98.2 \%$ identity at the nucleotide level, but FPV/ TR-SS/, reported from Turkey in 2010, showed less sequence homology, with $91.8 \%$.

$\mathrm{CPV}$, a ubiquitous pathogen worldwide, is a DNA virus that is amplified by host cell DNA polymerases, but has a high rate of genetic substitution, as in RNA 
viruses. This high rate helps in antigenic evolution, resulting in new variants (31). Despite this high substitution capacity, in this study a close nucleotide homology between the different CPV types was observed. The formation of parvovirus variants occurs through changes in the spike protein and determines host specificity (13). Between the variants, CPV-2c has a more eminent probability of evolution (3). In Europe, CPV-2a and CPV-2b are prevalent in some countries, but CPV-2c started to take the place of these antigenic variants, and in some others, CPV-2a was even not detected. $\mathrm{CPV}-2 \mathrm{c}$ is becoming more widespread and manifests itself (7).

In a recent study reported from Turkey, sequence analysis results from 25 different puppies between 1 and 8 months of age showed that CPV-2a compromises the majority of the variants and the CPV-2b strain was detected at a lower rate (2). In this study, all the field isolates obtained from symptomatic dogs were identified as CPV-2b strains. Phylogenetic analysis showed that although the acquired strains were clustered inside the CPV-2b clade, nucleotide homology analysis revealed that the mentioned strains are also

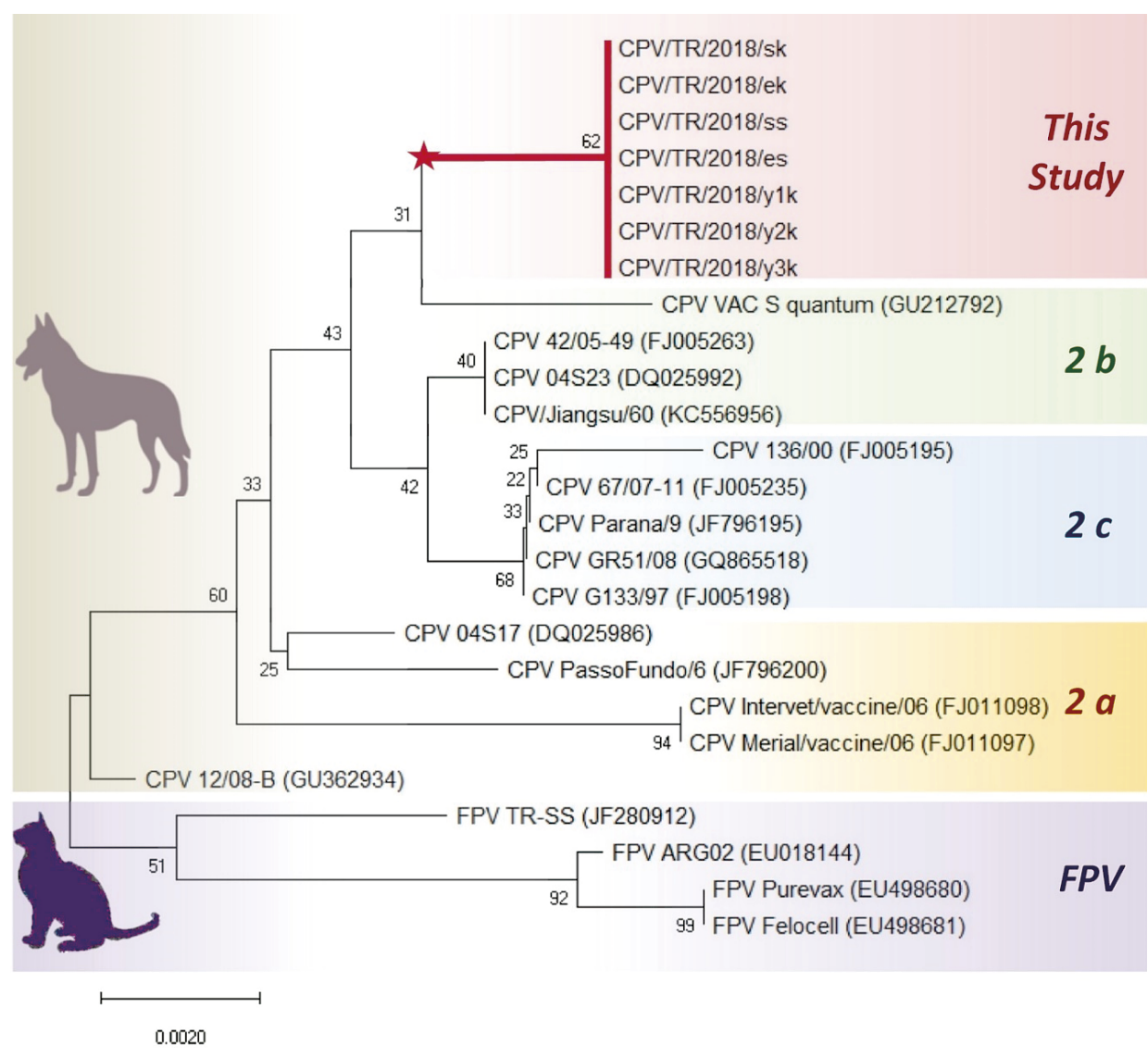

Fig. 3. Evolutionary relationships of taxa. The evolutionary history was inferred using the Neighbor-Joining method (38). The optimal tree with the sum of branch length $=\mathbf{0 . 0 3 7 0 8 8 2 0}$ is shown. The percentage of replicate trees in which the associated taxa clustered together in the bootstrap test (1000 replicates) are shown next to the branches (39). The tree is drawn to scale, with branch lengths in the same units as those of the evolutionary distances used to infer the phylogenetic tree. The evolutionary distances were computed using the Maximum Composite Likelihood method (40) and are in the units of the number of base substitutions per site. The analysis involved 25 nucleotide sequences. Codon positions included were $1^{\text {st }}+2^{\text {nd }}+3^{\text {rd }}+$ Noncoding. All ambiguous positions were removed for each sequence pair. There were a total of 378 positions in the final dataset. Evolutionary analyses were conducted in MEGA X (26) very close to the CPV-2a and CPV-2c strains. When compared to the CPV variants, the similarity of FPV strains appears to be lower, but they have a small difference and a high degree of similarity to our strains.
To understand the evolution of viruses and assume future changes in their genomes, molecular characterisation studies have a significant function. Their importance is seen in the distinction of different anti-
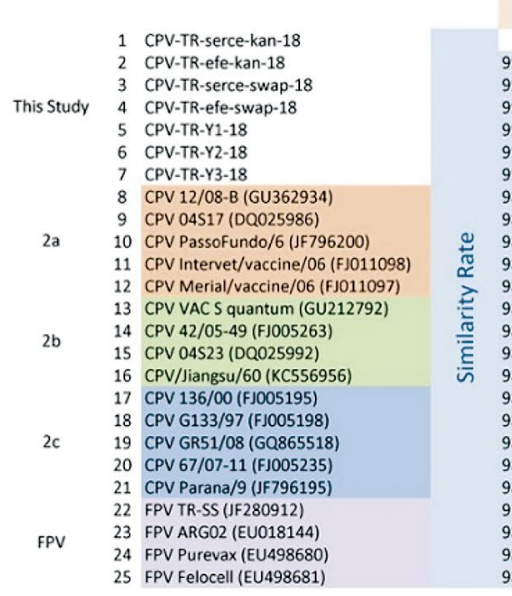

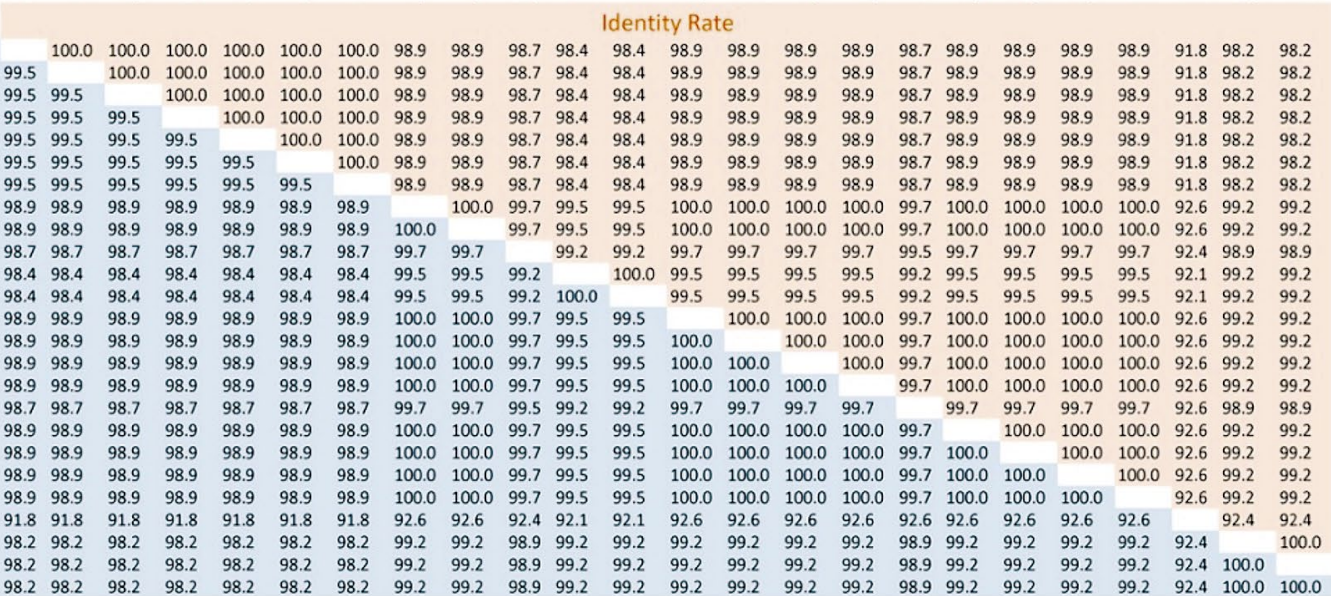

Fig. 4. Detailed nucleic acid identity and similarity rates for CPV/TR/2018/serçe/k CPV/TR/2018/efe/k, CPV/TR/2018/serçe/s, CPV/TR/2018/efe/s, CPV/TR/2018/Y1, CPV/TR/2018/Y2, and CPV/TR/2018/Y3 (Turkish) and 18 CPV and FPV nucleotide sequences used in phylogenetic tree shown in Fig. 3. 
genic types of CPV, which is a highly contagious viral disease of dogs, and in understanding the origins of the virus that is thought to have evolved from FPV. The CPV type 2 is no longer detectable in the field and the type $2 \mathrm{a}, 2 \mathrm{~b}$ and $2 \mathrm{c}$ variants have circulated instead. Notifications were made from Turkey regarding the CPV infections and although there are submissions of the 2c strains to the gene bank from Turkey, most circulating CPVs in dogs are the $2 \mathrm{a}$ and $2 \mathrm{~b}$ strains (22, $35,39)$. In this context, phylogenetic studies are needed to better understand the evolutionary relationships in the country in order to characterise the actual strains and elucidate the current situation.

Decaro et al. reported that the dogs vaccinated with type 2 or $2 b$ modified-live CPV vaccines shed a high amount of virus; shortly afterwards, the virus propagated in the blood and enteric membrane of immunised dogs (6). As in the world overall, in Turkey live vaccines against canine parvovirus are used for protection. In this study, viral DNA obtained from five different puppies shared a $100 \%$ nucleotide identity rate with each other and a $98.9 \%$ nucleotide identity with Quantum (vaccine) (2b) was observed. Two of the puppies were not socialised with each other or the other three puppies lived together. Although it is not known whether the animals were vaccinated or not, there is a possibility that the source of infection may have been caused by spreading from modified live vaccines or modified live-vaccinated dogs shedding $\mathrm{CPV}-2 \mathrm{~b}$ after vaccination.

CPV-2 is the prevalent cause of severe diarrhoea in puppies, even though neutralising antibodies can prevent CPV infection $(5,26)$. In some studies, nonvaccinated dogs demonstrated more sensitivity than vaccinated animals (12). It has also been shown that the prevalence of chronic gastrointestinal symptoms was higher in dogs who had previously had CPV infections (16). Therefore, in raising the living standards of dogs, vaccination, which is an effective method to reduce mortality by controlling dog parvovirus infection, is a measure that will make a difference $(5,23)$. When developing vaccination strategies, the right choice should be made to reduce the presence of the virus in the dog population by considering the viral spreading of live vaccines and different antigenic types. To ensure effective immunisation, vaccines should include the latest antigenic variants, and a strain that is able to cause disease even in vaccinated dogs, like CPV-2c, should also be considered $(5,38)$. To fully understand the impact of immunisation choices, prospective studies are needed.

Phylogenetic analysis of viruses causing gastroenteritis in dogs plays an important role in understanding their pathogenesis and epidemiology; it also supports diagnosis and treatment of the disease. Molecular characterisation results of CPV in diseased dogs demonstrated the presence of the $2 b$ strain in Turkey and provided up-to-date information about the infection status. As companion animals or for different purposes, the dog feeding rate is increasing in Turkey and there is limited literature on the parvoviruses of dogs. Therefore, studies on the elucidation of CPV infections and especially the genotypes circulating in dogs, which induce severe disease, provide the basis for future vaccines and will help understand the epidemiology of parvoviruses and develop new treatments.

\section{References}

1. Altman K., Kelman M., Ward M.: Are vaccine strain, type or administration protocol risk factors for canine parvovirus vaccine failure? Vet. Microbiol. 2017, 210, 8-16.

2. Appel M., Scott F., Carmichael L.: Isolation and immunisation studies of a canine parco-like virus from dogs with haemorrhagic enteritis. The Vet. Rec. 1979, 105, 156-159

3. Buonavoglia C., Martella V., PratelliA., Tempesta M., Cavalli A., BuonavogliaD., Bozzo G., Elia G., Decaro N., Carmichael L.: Evidence for evolution of canine parvovirus type 2 in Italy. J. Gen. Virol. 2001, 82, 3021-3025.

4. Cavalli A., Bozzo G., Decaro N., Tinelli A., Aliberti A., Buonavoglia D. Characterization of a canine parvovirus strain isolated from an adult dog. New Microbiol. 2001, 24, 239-242.

5. Decaro N., Buonavoglia C.: Canine parvovirus - a review of epidemiological and diagnostic aspects, with emphasis on type 2c. Vet. Microbiol. 2012, 155, $1-12$.

6.Decaro N., Crescenzo G., Desario C., Cavalli A., Losurdo M., Colaianni M. L., Ventrella G., Rizzi S., Aulicino S., Lucente M. S.: Long-term viremia and fecal shedding in pups after modified-live canine parvovirus vaccination. Vaccine 2014, 32, 3850-3853.

7. Decaro N., Desario C., Addie D. D., Martella V., Vieira M. J., Elia G., Zicola A., Davis C., Thompson G., Thiry E.: Molecular epidemiology of canine parvovirus, Europe. Emerg. Infect. Dis. 2007, 13, 1222.

8. Decaro N., Desario C., Elia G., Campolo M., Lorusso A., Mari V., Martella V., Buonavoglia $C .:$ Occurrence of severe gastroenteritis in pups after canine parvovirus vaccine administration: a clinical and laboratory diagnostic dilemma. Vaccine 2007, 25, 1161-1166.

9. Decaro N., Desario C., Elia G., Martella V., Mari V., Lavazza A., Nardi M., Buonavoglia $C$.: Evidence for immunisation failure in vaccinated adult dogs infected with canine parvovirus type 2c. New Microbiol. 2008, 31, 125-130.

10. Felsenstein J.: Confidence limits on phylogenies: an approach using the bootstrap. Evolution 1985, 39, 783-791.

11. Hong C., Decaro N., Desario C., Tanner P., Pardo M. C., Sanchez S. Buonavoglia C., Saliki J. T.: Occurrence of canine parvovirus type 2c in the United States. J. Vet. Diagn. Invest. 2007, 19, 535-539.

12. Houston D., Ribble C., Head L.: Risk factors associated with parvovirus enteritis in dogs: 283 cases (1982-1991). J. Am. Vet. Med. Assoc. 1996, 208, 542-546.

13. Hueffer K., Parrish C. R.: Parvovirus host range, cell tropism and evolution. Curr. Opin. Microbiol. 2003, 6, 392-398.

14. Ikeda Y., Mochizuki M., Naito R., Nakamura K., Miyazawa T., Mikami T., Takahashi E.: Predominance of canine parvovirus (CPV) in unvaccinated cat populations and emergence of new antigenic types of CPVs in cats. Virology 2002, 78, 13-19.

15. Ikeda Y., Nakamura K., Miyazawa T., Tohya Y., Takahashi E., Mochizuki M.: Feline host range of canine parvovirus: recent emergence of new antigenic types in cats. Emerg. Infect. Dis. 2002, 8, 341.

16. Kilian E., Suchodolski J. S., Hartmann K., Mueller R. S., Wess G., Unterer S. Long-term effects of canine parvovirus infection in dogs. PloS one 2018, 13, e0192198.

17. Kumar S., Stecher G., Li M., Knyaz C., Tamura K.: MEGA X: molecular evolutionary genetics analysis across computing platforms. Mol. Biol. Evol. 2018, 35, 1547-1549.

18. Martella V., Cavalli A., Pratelli A., Bozzo G., Camero M., Buonavoglia D., Narcisi D., Tempesta M., Buonavoglia C.: A canine parvovirus mutant is spreading in Italy. J. Clin. Microbiol. 2004, 42, 1333-1336.

19. Miranda C., Thompson G.: Canine parvovirus: the worldwide occurrence of antigenic variants. J. Gen. Virol. 2016, 97, 2043-2057.

20.Mochizuki M., San Gabriel M., Nakatani H., Yoshida M., Harasawa R.: Comparison of polymerase chain reaction with virus isolation and haemagglutination assays for the detection of canine parvoviruses in faecal specimens. Res. Vet. Sci. 1993, 55, 60-63. 
21. Muhire B. M., Varsani A., Martin D. P.: SDT: a virus classification tool based on pairwise sequence alignment and identity calculation. PloS one 2014, 9 , e108277.

22. Muz D., Oğuzoğlu T. C.., Timurkan M. Ö., Akın H.: Characterization of the partial VP2 gene region of canine parvoviruses in domestic cats from Turkey. Virus genes 2012, 44, 301-308.

23. Nandi S., Kumar M., Mohapatra T. K., Ravishankar C.: 2013. Emergence of canine parvovirus-2 variants and its impact on vaccination. World Appl. Sci. J. 23, 1366-1376.

24. Parrish C. R., Aquadro C. F., Strassheim M., Evermann J., SgroJ., Mohammed H. Rapid antigenic-type replacement and DNA sequence evolution of canine parvovirus. J. Virol. 1991, 65, 6544-6552.

25. Pereira C. A., Monezi T. A., Mehnert D. U., D'Angelo M., Durigon E. L.: Molecular characterization of canine parvovirus in Brazil by polymerase chain reaction assay. Vet. Microbiol. 2000, 75, 127-133.

26. Pratelli A., Cavalli A., Martella V., Tempesta M., Decaro N., Carmichael L. E., Buonavoglia C.: Canine parvovirus (CPV) vaccination: comparison of neutralizing antibody responses in pups after inoculation with CPV2 or CPV2b modified live virus vaccine. Clin. Diagn. Lab. Immunol. 2001, 8, 612-615.

27. Reed A. P., Jones E. V., Miller T. J.: Nucleotide sequence and genome organization of canine parvovirus. J. Virol. 1988, 62, 266-276.

28. Sahna K., Gencay A., Atalay O.: Viral aetiology of diarrhoea in puppies from a same shelter in Turkey: presence of mixed infections. Rev. Med. Vet. 2008, $159,345$.

29. Saitou N., Nei M.: The neighbor-joining method: a new method for reconstructing phylogenetic trees. Mol. Biol. Evol. 1987, 4, 406-425.

30. Sambrook J., Russell D. W., Russell D. W.: Molecular cloning: a laboratory manual (3-volume set). Cold spring harbor laboratory press New York, 2001
31. Shackelton L. A., Parrish C. R., Truyen U., Holmes E. C.: High rate of viral evolution associated with the emergence of carnivore parvovirus. Proc. Natl Acad. Sci. 2005, 102, 379-384

32. Steinel A., Parrish C. R., Bloom M. E., Truyen U.: Parvovirus infections in wild carnivores. J. Wildl. Dis. 2001, 37, 594-607.

33. Tamura K., Nei M., Kumar S.: Prospects for inferring very large phylogenies by using the neighbor-joining method. Proc. Natl. Acad. Sci. 2004, 101, 11030-11035.

34. Tattersall P., Bergoin M., Bloom M., Brown K., Linden R., Muzyczka N., Parrish C., Tijssen P.: Family parvoviridae. Virus Taxonomy: Eighth Report of the International Committee on Taxonomy of Viruses 2005, 353-369.

35. Timurkan M., Oğuzoğlu T.: Molecular characterization of canine parvovirus (CPV) infection in dogs in Turkey. Vet. Ital. 2015, 51, 39-44.

36. Truyen U., Evermann J. F., Vieler E., Parrish C. R.: Evolution of Canine Parvovirus Involved Loss and Gain of Feline Host Range. Virology 1996, 2, 186-189

37. Vela C., Cortés E., Simarro I., Casal J.: Identification of types of canine parvovirus circulating in Spain. Vet. Rec. 1995, 136, 174-175.

38. Woolford L., Crocker P., Bobrowski H., Baker T., Hemmatzadeh F.: Detection of the canine parvovirus 2c subtype in australian dogs. Viral Immunol. 2017, 30, 371-376.

39. Yilmaz Z., Pratelli A., Torun S.: Distribution of antigen types of canine parvovirus type 2 in dogs with hemorrhagic enteritis in Turkey. Turk. J. Vet. Anim. Sci. 2005, 29, 1073-1076.

Corresponding author: Dr. Zeynep Akkutay-Yoldar, DVM, PhD, Ankara University, Faculty of Veterinary Medicine, Department of Virology, 06110 , Ankara, Turkey; e-mail: akkutay@ankara.edu.tr 